\title{
Incidence and Mortality of Cancers Related to Secondhand Smoking in Southeast Asia Countries
}

\author{
Susi Ari Kristina ${ }^{1 *}$, Ni Putu Ayu Linda Permitasari², Kadek Ida Krisnadewi \\ Karina Anindita Santosa ${ }^{3}$
}

\begin{abstract}
Background: Secondhand smoke has been a big problem for human being worldwide as it is well-established risk factors for cancers. ASEAN (Association of Southeast Asian Nations) bear high burden of cancers since the high prevalence of secondhand smoke. The objective of this study is to estimate the burden of cancer attributable to secondhand smoking in ASEAN. Methods: This research was using descriptive epidemiological incidence and prevalence-based research design, with cancers incidence and mortality data gained from GLOBOCAN 2012. Secondhand smoke attributable fractions (SAFs) of six cancers (lung, bladder, colorectal, stomach, pancreas and larynx) were estimated and burden of cancers caused by secondhand smoking in ASEAN were calculated in term of incidence and mortality. Results: Secondhand smoking estimated for 453,562 cancer cases and 323,284 of total cancer mortality in 2012. The number of incidence and death of lung cancer attributable to secondhand smoking show the highest number compared with other type of cancers. Furthermore, we found that the number of cancer cases and cancer deaths attributable to secondhand smoking varied by each countries due to differences in size of population, various background risk of the cancer, and prevalence of secondhand smoking in each country. Conclusion: Secondhand smoking has been a risk factor for about two-fifth of cancer incidence and mortality in ASEAN. Therefore, ASEAN member countries are strongly encouraged to put in place stronger tobacco control policies and to strengthen the existing tobacco control measure in order to decrease the number of secondhand smokers and more effectively control cancers.
\end{abstract}

Keywords: Burden of disease- secondhand smoke- cancer- attributable fraction- ASEAN

Asian Pac J Cancer Prev, 20 (3), 971-976

\section{Introduction}

Secondhand smoke is a heterogeneous aerosol generated by burning tobacco. According to the study, there are 4000 harmful chemical compounds contained in cigarette smoke, including nicotine, tar, cyanide, benzene, cadmium, methanol, ammonia, and arsenic (World Health Organization, 2015). The amount of harmful substances in the body of secondhand smokers is greater than active smokers, because the toxins inhaled through the breath are not filtered; make them more susceptible to health problems compared to active smokers. On the other hand, although the prevalence rate of smoking among women is relatively low, women and children still have health risks as secondhand smokers due to men smoke at home or public space (Centers for Disease Control and Prevention, 2006).

Many studies have reported that exposure to secondhand smoke can cause a number of health problems (Centers for Disease Control and Prevention, 2006; Chen et al., 2017). Exposure to secondhand smoke is still a global health problem, because it can causes various diseases such as coronary heart disease, stroke, and respiratory disorders in adults, and infant death syndrome in infants and children (Chen et al., 2017). Several studies have also reported a link between cancers and secondhand smoking. From the International Agency for Research on Cancers' (IARC) monograph data, which is a summary of various meta-analysis of cancer due to secondhand smoking, shows the result that there is an association between secondhand smokers with some types of cancer indicated by relative risk value of more than one (World Health Organization, 2015).

In the other hand, secondhand smoke is a major problem in countries of the South-East Asia region as a large portion of the population smokes tobacco and the level of general awareness about the actual harmful effects of secondhand smoke is not sufficient (Sein et al., 2012; Kristina et al., 2015). The Association of Southeast Asian Nations (ASEAN) was established on 8 August 1967 in South East Asia region and consists of 10 countries namely Brunei Darussalam, Cambodia, 
Indonesia, Lao PDR, Malaysia, Myanmar, Philippines, Singapore, Thailand, and Vietnam. The member countries have a combined population of approximately 625 million people, accounted for $9 \%$ of the world's population (Lian and Dorotheo, 2013).

Like other places in the world, cancer is an increasing problem in ASEAN due to ageing and a transition to western lifestyle. It was recently estimated that there were over 700,000 new cases of cancer and 500,000 cancer deaths in ASEAN in the year 2012 (Kristina et al., 2016). Furthermore, these numbers were expected to increase, leading to substantial need for effective cancer control intervention and cancer service provision (Kimman et al., 2012; Kimman et al., 2015). Nevertheless, several ASEAN governments have been slow to react to this problem, leading to cancer epidemic in the region. Recent study indicated that over $75 \%$ of cancer patients in ASEAN experiencing death or financial catastrophic within one year. Based on the facts, ASEAN will bear burden of cancer due to secondhand smoking (Kimman et al., 2015).

However, there is currently no research on measuring the burden of cancer due to secondhand smoking in ASEAN. Given this background, the present study was performed to estimate the burden of cancers attributable to secondhand smoking in ASEAN member of countries. By assessing the estimated burden of cancer due to secondhand smoking, the data obtained can be used as a basis for further research and contribute in policy making in the context of prevention and control of tobacco and cigarettes through health promotion to raise public awareness about the negative impact of tobacco smoke, both in terms of the quality of life and the economic burden of the countries.

\section{Materials and Methods}

This research was using descriptive epidemiological incidence and prevalence-based research design to estimate the burden of cancers due to secondhand smoking in most member of ASEAN. There were 4 steps to gain the data; first we selected secondhand smoke-related cancers by systematic review. Second, we estimated population-related secondhand smoke attributable fraction (SAF) using relative risks and prevalence of secondhand smokers in each member of countries in ASEAN. Third, we calculated the number of incidence and cancer mortality of each country. Then lastly, we estimated smoking attributable incidence and mortality from the number of incidence and mortality multiplied by SAF.

\section{Secondhand smoking prevalence}

The prevalence of smoking was obtained from Global Adult Tobacco Survey (GATS) of each countries which reported by World Health Organization Regional Office for South East Asia. The data obtained mostly in 2011, for Cambodia, Indonesia, Malaysia and Thailand (Ministry of Health Malaysia, 2011; Ministry of Health Thailand, 2011; WHO Western Pasific Region, 2011; Ministry of Health Republic of Indonesia, 2012). Prevalence data from GATS for Myanmar was available in 2009 (Sein et al., 2012); Philippines and Viet Nam in 2015 (Ministry of Health Philippines, 2015; Ministry of Health Viet Nam, 2015). It should be noted that due to the data limitation, the prevalence data for Brunei Darussalam, Lao PDR and Singapore was unpublished. As shown in Table 1, the highest prevalence of secondhand smoking both among male and female was in Indonesia $(81.4 \%$ and $75.4 \%$ ), followed by Viet Nam for male (65.2\%), and Cambodia (53.5\%). Meanwhile, the second highest prevalence among female in ASEAN was Myanmar (57.8\%), followed by Viet Nam (55.0\%). On average, the prevalence of secondhand smoking was lowest among female than in male.

\section{Selection of secondhand smoke-related cancers}

The selection of secondhand smoke-related cancers included in this study was based on a systematic review. The relative risks for the cancers were obtained from monograph of meta-analysis by International Agency for Research on Cancers (IARC) of WHO (2015). According to the levels of evidence, we decided to include 6 secondhand smoke related cancer diseases which have RR value more than 1 and specifically got exposure in home. Those cancers are lung cancer (C33-34), colorectal (C18 - 21), stomach (C16), bladder (C23 - 24), pancreas (C25), and larynx (C32). Most relative risks (RRs) of secondhand smoking-related cancers were derived from recent many meta-analyses (World Health Organization, 2015), which consisted of research reported from many regions of the world. This global meta-analysis was selected because it is the most updated and that there is no meta-analysis conducted in Asia exists.

\section{Incidence and cancer mortality}

The number of cancer incidence cases and cancer mortality in ASEAN member countries in 2012 were derived from GLOBOCAN 2012 (IARC, 2012; Ferlay et al., 2013; Ferlay et al., 2015). GLOBOCAN estimated cancer incidence, mortality, and prevalence worldwide in 2012. The methods of estimation are country specific and the quality of the estimation depends upon the quality and on the amount of the information available for each country. Incidence data are derived from national cancer registries. If country data is not available, estimation by modeling, using incidence mortality ratios derived from recorded data in country or local cancer registries is used. Mortality statistics are based on national data that are collated and made available by the WHO for countries with vital registration. The cancer incidence and mortality of ASEAN member countries were shown in Table 2 and Table 3.

Estimation of secondhand smoke attributable fraction (SAF) of cancers

To calculate SAF values due to cancers, two parameters were considered: 1) the relative risks of secondhand smokers for the different related cancers, and 2) prevalence of secondhand smokers for male and female. The formula of SAF is shown below,

$$
\text { SAFs }=\frac{p(R R i-1)}{1+p(R R i-1)}
$$


where " $p$ " is the prevalence of secondhand smokers in the national population, "RR" is the relative risk of illness due to secondhand smoking, sub-script-i is a category of disease (World Health Organization, 2011). Exception for Singapore, due to there has been no any prevalence data of secondhand smoking, the SAF value gained and used directly from prevalence-based study of Cher et al., (2016) .

\section{Results}

Relative risks and SAF of countries members of ASEAN reported in Table 4. As shown in Table 4, secondhand smoke is attributable to about $33 \%-87 \%$ of lung cancer in ASEAN male meanwhile accounted to approximately $9 \%-71 \%$ in female.

Secondhand smoking attributable cancer incidence in ASEAN member countries has shown in Table 5. According to the estimation, secondhand smoking accounted for 453,562 new cancer cases in 2012 (303,525 male and 150,037 female cases). In male, the total number of secondhand smoking attributable cancer incidence was the highest in Viet Nam ( 76,173 cases), followed by Singapore $(48,020)$, and Thailand $(45,037$ cases $)$. On the other hand, the highest number of secondhand smoking attributable cancer incidence in female was found in Philippines $(44,767)$, followed by Viet Nam $(35,498)$ and Thailand $(17,241)$. In male, the number of patients with lung cancer attributable to secondhand smoking was the highest followed by colorectal cancer and stomach cancer. Meanwhile, in female, the number of patients with stomach cancer attributable to secondhand smoking was the highest followed by colorectal cancer and lung cancer.

In sum, total cancer mortality in ASEAN due to secondhand smoking accounted for 323,284 which consisted of 234,287 and 88,997 cancer mortality for male and female respectively as shown in Table 6 . When the
Table 1. Prevalence of Secondhand Smoking Among ASEAN Countries

\begin{tabular}{lccc}
\hline Country/year & $\begin{array}{c}\text { Number of } \\
\text { population } \\
\text { (million) }\end{array}$ & $\begin{array}{c}\text { Prevalence of secondhand } \\
\text { smoking }\end{array}$ \\
\hline Male (\%) & Female (\%) \\
\hline Indonesia/2011 & 14.5 & 53.5 & 33.3 \\
Malaysia/2011 & 241.9 & 81.4 & 75.4 \\
Myanmar/2009 & 50.1 & 53.0 & 33.3 \\
Philippines/2015 & 101.6 & 39.0 & 57.8 \\
Singapore/2010 & 5.0 & $\mathrm{NA}$ & NA \\
Thailand/2011 & 67.6 & 39.9 & 32.3 \\
Viet Nam/2015 & 91.7 & 65.2 & 55.0 \\
\hline
\end{tabular}

mortality is ranked by country, the total number of cancer death attributed by secondhand smoking was the highest in Viet Nam $(61,163)$, followed by Singapore $(39,850)$, and Thailand $(35,554)$. Among female population, the highest number of secondhand smoking attributable cancer mortality was found in Viet Nam $(22,884)$, followed by Singapore $(14,219)$ and Thailand $(13,278)$. In male, the number of patients with lung cancer mortality attributable to secondhand smoking was the highest $(128,049)$ followed by stomach cancer $(38,993)$, and colorectal cancer $(31,357)$. Similar pattern with male counterpart, the number of patients with lung cancer mortality attributable to secondhand smoking among female was the highest $(31,357)$ followed by stomach $(21,644)$ and colorectal cancer $(20,107)$.

\section{Discussion}

This study found that secondhand smoking was responsible for 453,562 cancer incidence in 2012 (303,525 male and 153,334 female cases), accounted for about

Table 2. Cancer Incidence in ASEAN Countries, 2012

\begin{tabular}{|c|c|c|c|c|c|c|c|c|c|c|}
\hline \multirow[t]{2}{*}{ Gender } & \multirow[t]{2}{*}{ Cancers } & \multicolumn{8}{|c|}{ Cancer incidence in ASEAN countries, 2012} & \multirow[t]{2}{*}{ Total } \\
\hline & & Cam & Ind & Mal & Mya & Phi & Sing & Thai & Viet & \\
\hline \multirow[t]{7}{*}{ Male } & Bladder & 128 & 5832 & 6,609 & 7,212 & 7,886 & 8,148 & 10,063 & 10,749 & 56,627 \\
\hline & Colorectal & 487 & 16,452 & 19,208 & 21,288 & 35,960 & 27,454 & 33,886 & 38,435 & 193,170 \\
\hline & Larynx & 82 & 2337 & 2,739 & 3,478 & 4,586 & 4,706 & 5,767 & 6,862 & 30,557 \\
\hline & Lung & 829 & 26,151 & 29,774 & 34,580 & 43,394 & 44,718 & 57,810 & 73,881 & 311,137 \\
\hline & Pancreas & 62 & 13,881 & 3,439 & 3,838 & 4,672 & 4,905 & 5,887 & 6,388 & 43,072 \\
\hline & Stomach & 324 & 4,135 & 5,369 & 8,478 & 9,884 & 10,286 & 11,896 & 21,292 & 71,664 \\
\hline & Total & 1,912 & 68,788 & 67,138 & 78,874 & 106,382 & 100,217 & 125,309 & 157,607 & 706,227 \\
\hline \multirow[t]{7}{*}{ Female } & Bladder & 78 & 1,350 & 1,541 & 1,808 & 2,118 & 2,194 & 2,810 & 3,025 & 14,924 \\
\hline & Colorectal & 420 & 12,207 & 14,354 & 16,133 & 20,008 & 21,176 & 26,231 & 50,509 & 161,038 \\
\hline & Larynx & 30 & 432 & 483 & 680 & 968 & 979 & 1,146 & 1,368 & 6,086 \\
\hline & Lung & 441 & 9,814 & 11,135 & 14,826 & 18,073 & 18,723 & 25,132 & 30,905 & 129,049 \\
\hline & Pancreas & 81 & 2,899 & 3,168 & 3,558 & 4,402 & 4,573 & 5,509 & 5,852 & 30,042 \\
\hline & Stomach & 206 & 2,405 & 3,169 & 4,973 & 59,799 & 6,224 & 7,455 & 12,249 & 96,480 \\
\hline & Total & 1,256 & 29,107 & 33,850 & 41,978 & 105,368 & 53,869 & 68,283 & 103,908 & 437,619 \\
\hline Total & & 3,168 & 97,895 & 100,988 & 120,852 & 211,750 & 154,086 & 193,592 & 261,515 & $1,143,846$ \\
\hline
\end{tabular}

Cam, Cambodia; Ind, Indonesia; Mal, Malaysia; Mya, Myanmar; Phi, Philippines; Sing, Singapore; Thai, Thailand; Viet, Vietnam. 
Table 3. Cancer Mortality in ASEAN Countries, 2012

\begin{tabular}{|c|c|c|c|c|c|c|c|c|c|c|}
\hline \multirow[t]{2}{*}{ Gender } & \multirow[t]{2}{*}{ Cancers } & \multicolumn{8}{|c|}{ Cancer mortality in ASEAN countries, 2012} & \multirow[t]{2}{*}{ Total } \\
\hline & & Cam & Ind & Mal & Mya & Phi & Sing & Thai & Viet & \\
\hline \multirow[t]{7}{*}{ Male } & Bladder & 76 & 3,016 & 3,276 & 3,652 & 3,944 & 3,999 & 4,975 & 5,342 & 28,280 \\
\hline & Colorectal & 349 & 10,908 & 12,323 & 13,876 & 16,579 & 17,128 & 20,968 & 24,080 & 116,211 \\
\hline & Larynx & 45 & 1,098 & 1,237 & 1,687 & 2,166 & 2,206 & 2,766 & 3,311 & 14,516 \\
\hline & Lung & 738 & 23,263 & 26,379 & 30,647 & 38,314 & 39,397 & 51,251 & 65,652 & 275,641 \\
\hline & Pancreas & 60 & 2,983 & 3,457 & 3,846 & 4,580 & 4,823 & 5,702 & 6,191 & 31,642 \\
\hline & Stomach & 296 & 3,726 & 4,302 & 7,220 & 8,415 & 8,677 & 9,972 & 18,563 & 61,171 \\
\hline & Total & 1,564 & 44,994 & 50,974 & 60,928 & 73,998 & 76,230 & 95,634 & 123,139 & 527,461 \\
\hline \multirow[t]{7}{*}{ Female } & Bladder & 47 & 706 & 761 & 927 & 1,065 & 1,085 & 1,397 & 1,516 & 7,504 \\
\hline & Colorectal & 289 & 8,142 & 9,295 & 10,627 & 12,825 & 13,220 & 16,228 & 19,092 & 89,718 \\
\hline & Larynx & 16 & 203 & 213 & 335 & 1,372 & 475 & 563 & 671 & 3,848 \\
\hline & Lung & 370 & 8,772 & 10,262 & 13,538 & 16,240 & 16,747 & 22,562 & 27,720 & 116,211 \\
\hline & Pancreas & 73 & 2,795 & 3,136 & 3,518 & 4,260 & 4,468 & 5,311 & 5,643 & 29,204 \\
\hline & Stomach & 183 & 2,165 & 2,555 & 4,248 & 5,096 & 5,265 & 6,256 & 10,596 & 36,364 \\
\hline & Total & 183 & 22,783 & 26,222 & 33,193 & 40,858 & 41,260 & 52,317 & 65,238 & 282,054 \\
\hline Total & & 1,747 & 67,777 & 77,196 & 94,121 & 114,856 & 117,490 & 147,951 & 188,377 & 809,515 \\
\hline
\end{tabular}

Cam, Cambodia; Ind, Indonesia; Mal, Malaysia; Mya, Myanmar; Phi, Philippines; Sing, Singapore; Thai, Thailand; Viet, Vietnam.

$39.65 \%$ of the new cases of cancer in ASEAN in 2012. In term of mortality, secondhand smoking was accounted for $44.42 \%$ of cancer deaths in male $(234,287)$ and $31.55 \%$ deaths in female $(96,265)$. Based on the types of cancer, the strongest association with secondhand smoking was found for lung cancer and it is also responsible for the highest number of death both in men and women compared with other type of cancer significantly.

Exposure to secondhand smoke has been identified as a risk factor for various cancers associated with the respiratory system, especially lung cancer (Hori et al., 2016), since the last three decades. In Asomaning et al., (2008) research, it was reported that people exposed to secondhand smoke have a higher risk of lung cancer than active smokers, especially in subjects exposed to tobacco smoke before the age of 25 years. Tobacco smoke particles that accumulate in the lungs through the respiratory system can lead to sister chromosome exchange, DNA oxidative damage, and increase the number of P53 mutations in lung cancer.

Previous meta-analysis revealed a statistically significant association between secondhand smoke exposure and lung cancer in Japanese non-smokers. According to the National Health and Nutrition Survey in Japan 2013, 20.6\% people were exposed to secondhand smoke at least once a month at home with the overall relative risk of lung cancer from secondhand smoke exposure is about 1.3. That meta-analysis confirmed a significant association between secondhand smoking and lung cancer has a critical implication for promoting legislation to prevent secondhand smoke exposure and enact comprehensive tobacco control policies in Japan,

Table 4. Relative Risks and Secondhand Smoking Attributable Fractions (SAFs) of Selected Cancers in ASEAN Countries, 2012

\begin{tabular}{llccccccccc}
\hline Gender & Cancers & RR & \multicolumn{7}{c}{ Secondhand smoking attributable fractions (SAF) } \\
& & & Cam & Ind & Mal & Mya & Phi & Sing & Thai & Viet \\
\hline Male & Bladder & 1.75 & 0.29 & 0.38 & 0.25 & 0.28 & 0.23 & 0.36 & 0.23 & 0.33 \\
& Colorectal & 1.9 & 0.33 & 0.42 & 0.28 & 0.32 & 0.26 & NA & 0.26 & 0.37 \\
& Larynx & 2.9 & 0.5 & 0.61 & 0.45 & 0.5 & 0.43 & 0.69 & 0.43 & 0.55 \\
& Lung & 2.28 & 0.41 & 0.51 & 0.36 & 0.4 & 0.33 & 0.87 & 0.34 & 0.45 \\
& Pancreas & 6.0 & 0.73 & 0.8 & 0.68 & 0.72 & 0.66 & 0.2 & 0.67 & 0.77 \\
& Stomach & 5.8 & 0.72 & 0.8 & 0.68 & 0.71 & 0.65 & 0.19 & 0.66 & 0.76 \\
& Bladder & 3.3 & 0.43 & 0.63 & 0.43 & 0.57 & 0.41 & 0.41 & 0.43 & 0.56 \\
& Colorectal & 1.8 & 0.21 & 0.38 & 0.21 & 0.32 & 0.2 & NA & 0.21 & 0.31 \\
& Larynx & 2.9 & 0.39 & 0.59 & 0.39 & 0.52 & 0.37 & 0.66 & 0.38 & 0.51 \\
& Lung & 1.31 & 0.09 & 0.19 & 0.09 & 0.15 & 0.09 & 0.71 & 0.09 & 0.15 \\
& Pancreas & 6.0 & 0.62 & 0.79 & 0.62 & 0.74 & 0.6 & 0.21 & 0.62 & 0.73 \\
& Stomach & 5.8 & 0.62 & 0.78 & 0.62 & 0.74 & 0.59 & 0.12 & 0.61 & 0.73 \\
\hline
\end{tabular}

Cam, Cambodia; Ind, Indonesia; Mal, Malaysia; Mya, Myanmar; Phi, Philippines; Sing, Singapore; Thai, Thailand; Viet, Vietnam. 
Table 5. Secondhand Smoking Attributable Cancer Incidence in ASEAN Countries, 2012

\begin{tabular}{|c|c|c|c|c|c|c|c|c|c|c|}
\hline \multirow[t]{2}{*}{ Gender } & \multirow[t]{2}{*}{ Cancers } & \multicolumn{8}{|c|}{ Secondhand smoking incidence (2012) } & \multirow[t]{2}{*}{ Total } \\
\hline & & Cam & Ind & Mal & Mya & Phi & Sing & Thai & Viet & \\
\hline \multirow[t]{7}{*}{ Male } & Bladder & 37 & 2,211 & 1,620 & 2,024 & 1,785 & 2,933 & 2,318 & 3,530 & 16,458 \\
\hline & Colorectal & 158 & 6,956 & 5,386 & 6,787 & 9,343 & NA & 8,953 & 14,213 & 51,796 \\
\hline & Larynx & 41 & 1,419 & 1,236 & 1,729 & 1,952 & 3,247 & 2,487 & 3,797 & 15,908 \\
\hline & Lung & 337 & 13,344 & 10,617 & 13,819 & 14,449 & 38,905 & 19,543 & 33,609 & 144,623 \\
\hline & Pancreas & 45 & 11,143 & 2,352 & 2,772 & 3,088 & 981 & 3,921 & 4,888 & 29,190 \\
\hline & Stomach & 233 & 3,292 & 3,625 & 6,053 & 6,442 & 1,954 & 7,815 & 16,136 & 45,550 \\
\hline & Total & 851 & 38,365 & 24,836 & 33,184 & 37,059 & 48,020 & 45,037 & 76,173 & 303,525 \\
\hline \multirow[t]{7}{*}{ Female } & Bladder & 34 & 856 & 668 & 1,032 & 870 & 900 & 1,198 & 1,689 & 7,247 \\
\hline & Colorectal & 88 & 4,593 & 3,020 & 5,101 & 3,904 & NA & 5,386 & 15,433 & 37,525 \\
\hline & Larynx & 12 & 254 & 187 & 356 & 354 & 656 & 436 & 699 & 2,954 \\
\hline & Lung & 41 & 1,859 & 1,042 & 2,253 & 1,552 & 13,293 & 2,287 & 4,502 & 26,829 \\
\hline & Pancreas & 51 & 2,291 & 1,979 & 2,643 & 2,652 & 960 & 3,402 & 4,291 & 18,269 \\
\hline & Stomach & 127 & 1,884 & 1,949 & 3,655 & 35,435 & 747 & 4,532 & 8,884 & 57,213 \\
\hline & Total & 353 & 11,737 & 8,845 & 15,040 & 44,767 & 16,565 & 17,241 & 35,498 & 150,037 \\
\hline Total & & 1,204 & 50,102 & 33,681 & 48,224 & 81,826 & 64,576 & 62,278 & 111,671 & 453,562 \\
\hline
\end{tabular}

Cam, Cambodia; Ind, Indonesia; Mal, Malaysia; Mya, Myanmar; Phi, Philippines; Sing, Singapore; Thai, Thailand; Viet, Vietnam.

as one of states in Asia Pacific Region (Hori et al., 2016).

Furthermore, although the exposure to tobacco smoke is very high from public places, the duration of the exposure is not proportional to the time at home or workplace where people often spend most of their time. Therefore, appropriate policies to reduce both home and workplace smokers are also indispensable. Not only the ban or punishment, appreciation may also need to be given to the agency or workplace that is firmly in the smoking ban. To effectively reduce the incidence of secondhand smokers at home, it is necessary to counsel, educate, and advise on the dangers of tobacco smoke and make plans to eliminate the habits, especially in men (Asomaning et al., 2008; Hori et al., 2016; Zahra et al., 2016).
The other study conducted in Korea, as another country in Asia Pacific, reported 68\% of nonsmokers were exposed to secondhand smoke during a typical day. The results highlight the need for strong, comprehensive secondhand smoking control measures, such as a complete ban of smoking in all workplaces and public places, as well as public health campaigns to promote home smoking bans and non-smoking norms. That study said, to prevent workplace exposure for all Koreans, the law should be expanded to a complete ban on smoking in all workplaces, and should provide for enforcement measures and smoking cessation programs (Hughes et al., 2008).

When looking at each countries in ASEAN, we found that the number of cancer cases and cancer

Table 6. Secondhand Smoking Attributable Cancer Mortality in ASEAN Countries, 2012

\begin{tabular}{|c|c|c|c|c|c|c|c|c|c|c|}
\hline \multirow[t]{2}{*}{ Gender } & \multirow[t]{2}{*}{ Cancers } & \multicolumn{8}{|c|}{ Secondhand smoking mortality (2012) } & \multirow[t]{2}{*}{ Total } \\
\hline & & Cam & Ind & Mal & Mya & Phi & Sing & Thai & Viet & \\
\hline \multirow[t]{7}{*}{ Male } & Bladder & 22 & 1,143 & 803 & 1,025 & 893 & 1,440 & 1,146 & 1,754 & 8,226 \\
\hline & Colorectal & 113 & 4,612 & 3,456 & 4,424 & 4,307 & NA & 5,540 & 8,905 & 31,357 \\
\hline & Larynx & 23 & 667 & 558 & 838 & 922 & 1,522 & 1,193 & 1,832 & 7,555 \\
\hline & Lung & 300 & 11,870 & 9,407 & 12,247 & 12,758 & 34,275 & 17,326 & 29,866 & 128,049 \\
\hline & Pancreas & 44 & 2,395 & 2,365 & 2,778 & 3,027 & 965 & 3,798 & 4,738 & 20,110 \\
\hline & Stomach & 213 & 2,967 & 2,905 & 5,155 & 5,485 & 1,649 & 6,551 & 14,068 & 38,993 \\
\hline & Total & 715 & 23,654 & 19,493 & 26,466 & 27,392 & 39,850 & 35,554 & 61,163 & 234,287 \\
\hline \multirow[t]{7}{*}{ Female } & Bladder & 20 & 448 & 330 & 529 & 437 & 445 & 595 & 847 & 3,651 \\
\hline & Colorectal & 61 & 3,063 & 1,955 & 3,360 & 2,502 & NA & 3,332 & 5,834 & 20,107 \\
\hline & Larynx & 6 & 120 & 83 & 175 & 501 & 314 & 214 & 343 & 1,756 \\
\hline & Lung & 35 & 1,662 & 960 & 2,057 & 1,394 & 11,890 & 2,054 & 4,038 & 31,357 \\
\hline & Pancreas & 46 & 2,209 & 1,959 & 2,614 & 2,566 & 938 & 3,280 & 4,138 & 17,750 \\
\hline & Stomach & 113 & 1,696 & 1,572 & 3,123 & 3,020 & 632 & 3,803 & 7,685 & 21,644 \\
\hline & Total & 280 & 9,198 & 6,859 & 11,858 & 10,421 & 14,219 & 13,278 & 22,884 & 88,997 \\
\hline Total & & 995 & 32,852 & 26,352 & 38,324 & 37,813 & 54,069 & 48,832 & 84,047 & 323,284 \\
\hline
\end{tabular}

Cam, Cambodia; Ind, Indonesia; Mal, Malaysia; Mya, Myanmar; Phi, Philippines; Sing, Singapore; Thai, Thailand; Viet, Vietnam. 
deaths attributable to secondhand smoking varied by each countries. The highest secondhand smoking attributable cancer mortality was in Viet Nam $(84,047)$, Singapore $(54,069)$ and Thailand $(48,832)$ respectively. Meanwhile, the deaths attributable to secondhand smoking in Cambodia was the lowest, only 995. These can be explained by difference in size of population, various background risk of the cancer, and prevalence of secondhand smoking in each country.

In many studies, there are risk factors other than tobacco smoke associated with cancers such as diet, body mass index (BMI), and alcohol consumption (Peppone et al., 2009; Peppone et al., 2010) are not included in the research model and thus have the potential to cause bias towards the results of the study, included in this study which is became one of our limitations. Secondly, RRs used in our analysis were not limited to ASEAN population. Further study examining the risk of cancer related to secondhand smoking should be conducted among ASEAN population for more valid estimation. Nevertheless, due to the lack data of secondhand smoking prevalence in some counties of ASEAN, cancer incidence and mortality attributable by secondhand smoking in this study just conducted for 8 countries (without Brunei Darussalam and Lao PDR). The prevalence of secondhand smoke data from each country of ASEAN member came from different year, it might influences the results. Furthermore, due to substantial economic development in ASEAN might be changed dramatically so that the SAF may need to be re-estimated.

Based on our study, secondhand smoking accounted for 453,562 new cancer cases and 323,284 of total cancer mortality in 2012 with varied number by each country due to differences in size of population, various background risk of the cancer, and prevalence of secondhand smoking in each country. As many ASEAN countries are in the early stage of the tobacco epidemic, it is likely that the burden of cancer attributable to secondhand smoking will continue to rise over the next decades. Therefore, ASEAN member countries are strongly encouraged to put in place stronger tobacco control policies and to strengthen the existing tobacco control measure in order to effectively control cancer, as the World Health Organization (WHO) Framework Convention on Tobacco Control (FCTC) (World Health Organization, 2009) recommends comprehensive smoke-free policies to protect people from secondhand smoke.

\section{Conflict of interest}

The authors declare there is no any conflict of interest.

\section{References}

Asomaning K, Miller DP, Liu G, et al (2008). Second hand smoke, age of exposure and lung cancer risk. J Lung Cancer, 61, 13-20.

Chen J, Wang MP, Wang X, et al (2017). Secondhand smoke exposure (SHS) and health-related quality of life (HRQoL) in Chinese never smokers in Hong Kong. BMJ Open, 5 , e007694.

Cher BP, Chen C, Yoong J (2016). Prevalence-based, disease-specific estimate of the social cost of smoking in
Singapore. BMJ Open, 7, e014377.

Ferlay J, Soerjomataram I, Dikshit R, et al (2013). Cancer incidence and mortality worldwide : IARC Cancer Base No. 11 [Online]. Available: globocan.iarc.fr [Accessed 27 February 2018].

Ferlay J, Soerjomataram I, Dikshit R, et al (2015). Cancer incidence and mortality worldwide: sources, methods and major patterns in GLOBOCAN 2012. Int J Cancer, 136, 359-86.

Hori M, Tanaka H, Wakai K, et al (2016). Secondhand smoke exposure and risk of lung cancer in Japan: a systematic review andmeta-analysis of epidemiologic studies. Jpn J Clin Oncol, 46, 10.

Hughes SC, Corcos IA, Hofstetter CR, et al (2008). Secondhand smoke exposure among nonsmoking adults in Seoul, Korea. Asian Pacific J Cancer Prev, 9, 247-52.

Kimman M, Jan S, Yip CH, et al (2015). Catastrophic health expenditure and 12-month mortality associated with cancer in Southeast Asia: results from a longitudinal study in eight countries. BMC Med, 13, 190.

Kimman M, Norman R, Kingston M, et al (2012). The burden of cancer in member countries of the Association of Southeast Asian Nations (ASEAN). Asian Pac J Cancer Prev, 13, 411-20.

Kristina SA, Endarti D, Thavorncharoensap M (2016). Burden of cancer attributable to tobacco smoking in member countries of the Association of Southeast Asian Nations (ASEAN), 2012. Cancer Epidemiol, 44, 84-90.

Kristina SA, Thavorncharoensap M, Pongcharoensuk P, et al (2015). Impact of smoking cessation training for community pharmacists in Indonesia. Asian Pac J Cancer Prev, 16, 3319-23.

Peppone LJ, Hyland A, Moysich KB, et al (2009). Examining the association between cigarette smoking and colorectal cancer using historical case-control data. Cancer Epidemiol, 33, $182-8$

Peppone LJ, Reid ME, Moysich KB, et al (2010). The effect of secondhand smoke exposure on the association between active cigarette smoking and colorectal cancer. Cancer Causes Control, 21, 1247-55.

Sein AA, Than-Htike MM, Sinha DN, et al (2012). Exposure to secondhand tobacco smoke among adults in Myanmar. Indian J Cancer, 49, 410-8.

Zahra A, Cheong HK, Lee EW, et al (2016). Burden of Disease Attributable to Secondhand Smoking in Korea. Asia Pac J Public Health, 1, 14.

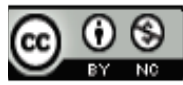

This work is licensed under a Creative Commons AttributionNon Commercial 4.0 International License. 